\title{
Development and Validation of a Reduced DME Mechanism Applicable to Various Combustion Modes in Internal Combustion Engines
}

\author{
Gregory T. Chin, J.-Y. Chen, Vi H. Rapp, and R. W. Dibble \\ Department of Mechanical Engineering, University of California at Berkeley, Berkeley, CA 94720, USA \\ Correspondence should be addressed to Gregory T. Chin, gchin@berkeley.edu
}

Received 19 October 2010; Revised 7 March 2011; Accepted 28 March 2011

Academic Editor: A. K. Gupta

Copyright ( $) 2011$ Gregory T. Chin et al. This is an open access article distributed under the Creative Commons Attribution License, which permits unrestricted use, distribution, and reproduction in any medium, provided the original work is properly cited.

\begin{abstract}
A 28-species reduced chemistry mechanism for Dimethyl Ether (DME) combustion is developed on the basis of a recent detailed mechanism by Zhao et al. (2008). The construction of reduced chemistry was carried out with automatic algorithms incorporating newly developed strategies. The performance of the reduced mechanism is assessed over a wide range of combustion conditions anticipated to occur in future advanced piston internal combustion engines, such as HCCI, SAHCCI, and PCCI. Overall, the reduced chemistry gives results in good agreement with those from the detailed mechanism for all the combustion modes tested. While the detailed mechanism by Zhao et al. (2008) shows reasonable agreement with the shock tube autoignition delay data, the detailed mechanism requires further improvement in order to better predict HCCI combustion under engine conditions.
\end{abstract}

\section{Introduction}

Recently, interest in developing new fuels and technologies for cleaner, more efficient, and environmentally sustainable combustion has grown. Dimethyl ether (DME) is considered a promising alternative fuel since it can be synthesized from emerging renewable energy sources, such as biomass, as well as existing fossil fuel sources [1]. More importantly, DME possesses favorable combustion characteristics. DME has a high cetane number, a low auto-ignition temperature, and its combustion products contain less harmful emissions relative to diesel fuel. That is, the exhaust from the combustion of DME has low levels of particulates, $\mathrm{CO}, \mathrm{NOx}$, and contains no sulphur-containing products. These positive features make DME a good candidate for replacing diesel fuel under Compression Ignition (CI) $[1,2]$. The potential use of DME can be extended to emerging engine technologies, such as Homogenous Charge Compression Ignition (HCCI), Spark Assisted HCCI (SAHCCI), and Partial Charge Compression Ignition (PCCI).

DME combustion has been the subject of numerous experimental and numerical studies. A shock tube study was conducted by Pfahl et al. [3] in which the auto-ignition delays of DME-air mixtures were measured over a range of pressures and temperatures. Dagaut et al. [4] studied the kinetics of DME in a jet-stirred reactor and developed a DME kinetics model for high temperatures. Later, another DME chemical mechanism containing 78 species was developed by Curran et al. [5] and the mechanism was validated with the shock tube data by Pfahl et al. [3] and the jet-stirred reactor by Dagaut et al. [4]. Dagaut et al. [6] expanded on the Curran et al. [5] DME mechanism by including low-temperature pathways. The updated mechanism contained 55 species and was validated with jet-stirred reactor data and hightemperature shock tube data. Fischer et al. [7] and Curran et al. [8] carried out joint studies on high-temperature and low-temperature oxidation of DME, respectively. In these two papers, experimental data was used to further refine the DME mechanism developed by Curran et al. [5]. In the paper by Kaiser et al. [9], species measurements were performed on a DME flat flame at standard pressure and measurements were compared to a numerical simulation utilizing the detailed mechanism refined by Fischer et al. [7]. Recently, a compact DME detailed mechanism was developed by 
TABLE 1: List of QSS Species.

\begin{tabular}{llll}
\hline $\mathrm{OCH}_{2} \mathrm{OCHO}$ & $\mathrm{HCCOH}$ & $\mathrm{HOCH}_{2} \mathrm{O}$ & $\mathrm{OCHO}$ \\
$\mathrm{C}_{2} \mathrm{H}$ & $\mathrm{C}_{2} \mathrm{H}_{3}$ & $\mathrm{CH}_{3} \mathrm{OCH}_{2}$ & $\mathrm{CH}_{3} \mathrm{O}$ \\
$\mathrm{CH}_{2} \mathrm{OH}$ & $\mathrm{CH}_{2} \mathrm{~S}$ & $\mathrm{CH}_{3} \mathrm{OCH}_{2} \mathrm{O}$ & $\mathrm{CH}_{3} \mathrm{OCO}$ \\
$\mathrm{CH}_{3} \mathrm{CO}$ & $\mathrm{CH}_{2} \mathrm{OCH}_{2} \mathrm{O}_{2} \mathrm{H}$ & $\mathrm{O}_{2} \mathrm{CH}_{2} \mathrm{OCH}_{2} \mathrm{O}_{2} \mathrm{H}$ & $\mathrm{C}_{2} \mathrm{H}_{4} \mathrm{OH}$ \\
$\mathrm{HOC}_{2} \mathrm{H}_{4} \mathrm{O}_{2}$ & $\mathrm{CH}_{3} \mathrm{CH}_{2} \mathrm{O}$ & $\mathrm{C}_{2} \mathrm{H}_{5} \mathrm{OH}$ & $\mathrm{CH}_{3} \mathrm{CHOH}$ \\
$\mathrm{HCO}$ & $\mathrm{CH}_{2} \mathrm{CO}$ & $\mathrm{CH}_{2} \mathrm{HCO}$ & $\mathrm{HCCO}$ \\
$\mathrm{CH}_{3} \mathrm{HCO}$ & & & \\
\hline
\end{tabular}

Zhao et al. [10] in which an updated high-temperature model was coupled to the low-temperature model developed by Fischer et al. [7].

In parallel to the development of detailed DME chemistry, several attempts have been made in the construction of simplified DME mechanisms. Based on the detailed mechanism by Curran et al. [8] and Yamada et al. [11] constructed a 23-species DME skeletal chemistry model for CI applications by removing unimportant reactions and species. Using the simplified chemistry model developed by Liang [12] for DME/methanol fuel mixtures, Yao et al. [13] simulated the combustion of a DME/methanol fuel mixture in a HCCI engine. Based on the detailed mechanisms developed by Fischer et al. [7] and Curran et al. [8], Yao et al. [14, 15] developed a 26-species skeletal mechanism for use in high compression HCCI engines. Recently, Huang et al. [16] performed a multidimensional simulation for a singlecylinder, direct-injection HCCI engine based on the 26species skeletal chemistry by Yao et al. [14, 15].

The purpose of developing simplified mechanisms is to save computational resources while accurately capturing the main combustion features under the regime of study. Therefore, validation of a simplified chemistry mechanism needs to be thoughtfully performed to ensure accuracy and reliability of the solution. The present study aims to develop an accurate simplified chemistry mechanism over a variety of combustion regimes. The intended application of the mechanism is not only limited to CI mode but also includes HCCI, SAHCCI, and PCCI combustion modes. The paper is organized as follows. First, the simplified mechanism, known simply as the "reduced" chemistry, is developed through the Quasi-Steady State (QSS) approach which has been coupled with other advanced numerical strategies. Second, the performance of the reduced chemistry is extensively assessed for various combustion modes representative of CI, HCCI, SAHCCI, and PCCI engines. Third, application of the reduced chemistry to HCCI engine simulations is performed and the numerical results are compared to measured data. Lastly, conclusions are drawn and comments regarding further improvements to the DME mechanisms are made.

\section{Development of Simplified Chemistry}

In the numerical simulation of combustion systems, the computational time of combustion chemistry scales roughly
TABLE 2: Targeted conditions used for development of reduced chemistry: autoignition at constant pressure.

\begin{tabular}{lcc}
\hline $\begin{array}{l}\text { Equivalence Ratio } \\
(\phi)\end{array}$ & $\begin{array}{c}\text { Initial Temperature } \\
(\mathrm{K})\end{array}$ & $\begin{array}{c}\text { Initial Pressure } \\
(\mathrm{atm})\end{array}$ \\
\hline 0.5 & 950 & 1 \\
1 & 950 & 1 \\
3 & 950 & 1 \\
0.5 & 800 & 10 \\
1 & 800 & 10 \\
3 & 800 & 10 \\
\hline
\end{tabular}

with $N_{s}^{2}$, where $N_{s}$ is the total number of species in the reaction mechanism. Therefore decreasing $N_{s}$ can significantly save computing resources. Two types of simplified chemistry models can be developed from a detailed mechanism: (1) a skeletal mechanism and (2) a reduced mechanism. A skeletal mechanism is obtained by removing species and reaction steps unimportant to the combustion regime of interest from the detailed chemistry, such as the 23-species skeletal mechanism developed by Yamada et al. [11]. Although adjusting certain controlling rate constants in a skeletal mechanism is often done to improve its performance for a narrow combustion regime, such an empirical approach has limited applications. On the basis of a validated skeletal mechanism, further reduction in the total number of species can be made by assuming QSS for certain intermediate species. These QSS species are evaluated by their own algebraic equations and therefore do not appear in the species list.

Due to the limitations of the previously developed skeletal mechanisms to a narrow combustion regime, it was decided to develop a new reduced mechanism that would achieve the goal of being applicable for a wide variety of combustion modes. Since the detailed DME mechanism by Zhao et al. [10] contains only 55 species, the mechanism is small enough for development of reduced chemistry directly using the QSS approach. By properly identifying 25 QSS intermediate species and omitting Argon and Helium, the present reduced chemistry can be reduced to 28 species. These 25 QSS species, listed in Table 1, were identified using the Targeted Search Algorithm (TSA) developed recently by Tham et al. [17]. TSA dictates that during the construction phase of a reduced mechanism, the reduced system is immediately tested under representative conditions to ensure its overall accuracy. As a result, the impact of a group of QSS species on the predicted accuracy is assessed immediately in the construction phase and used to guide the selection of the appropriate group of QSS species. One important advantage of this approach is its potential to significantly reduce the size of a complex mechanism while ensuring its predictive capabilities for the targeted regime, both accomplished in a systematic manner. Table 2 lists the targeted conditions for autoignition used during the TSA process. An additional targeted condition for rich premixed flames (equivalence ratio of 1.1, initial temperature of $300 \mathrm{~K}$, and an initial pressure of $1 \mathrm{~atm}$ ) was also included. HCCI engine cases were not included in the list of targeted 
conditions as the added computational cost of a singlezone model coupled with TSA would make the approach too computationally intensive. However, past experiences with an isooctane mechanism [17] have suggested that as long as the targeted ignition conditions are properly chosen to represent critical conditions in HCCI engines, the resulting reduced mechanism is satisfactory. TSA was used to identify suitable QSS species such that the resulting reduced chemistry would predict ignition delays and flame speeds within $10 \%$ of those predicted with the detailed mechanism. The entire process was automated using a computer by coupling the TSA with the Computer-Assisted Reduction Mechanism (CARM) algorithm [18]. The combined fixedpoint iteration and matrix inversion algorithm [19] was used for accurate and quick calculations of the QSS species concentrations. Please refer to the supplementary material available online at doi:101155/2011/630580 for a copy of the developed 28-species reduced chemistry.

\section{Results and Discussions}

This section evaluates the performance of the 28-species DME reduced chemistry mechanism through comparisons against the detailed mechanism by Zhao et al. [10] over a variety of combustion conditions. As mentioned above, it is the goal of the present reduced mechanism to be applicable not only to CI engines but also to HCCI, PCCI, and SAHCCI engines as well. The following combustion conditions were chosen to test the effectiveness of the reduced mechanism over the wide range of combustion modes found in these engines.

3.1. Autoignition Delays. The auto-ignition delays of a stochiometric DME and air mixture were computed over a range of temperatures $(650 \mathrm{~K}-1300 \mathrm{~K})$ at initial pressures of $13 \mathrm{bar}$ and 40 bar using the Sandia code SENKIN [20]. These conditions correspond to those in the shock tube study by Pfahl et al. [3]. Using the detailed mechanism by Zhao et al. [10] and the present 28-species reduced chemistry, computations were carried out at constant volume to simulate ignition in a shock tube. Additional calculations were conducted with the 26-species skeletal mechanism by Yao et al. $[14,15]$ for further comparisons.

Figure 1 compares the computed ignition delays with the three mechanisms discussed above against the shock tube data by Pfahl et al. [3]. An uncertainty in the initial shock tube pressure of 1.5 bar and 2 bar is reported by Pfahl et al. [3] for the 13 bar and 40 bars shock tube data, respectively. [3]. At low temperatures $(650 \mathrm{~K}$ to $800 \mathrm{~K})$ and at 40 bar, the computed delays from all three mechanisms are slightly longer than the shock tube data. However within the same temperature range at 13 bars, the 26-species skeletal mechanism by Yao et al. $[14,15]$ predicted delays much longer than those by the detailed mechanism of Zhao et al. [10] and the present 28-species chemistry. The present 28 -species chemistry (short dashed lines) predicted slightly lower ignition delay than the detailed chemistry (long dot-dashed lines) [10]. At high temperatures above

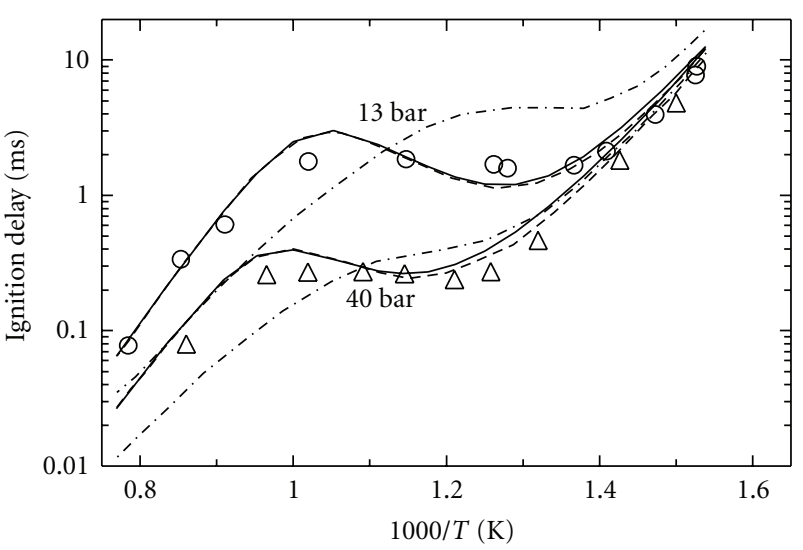

FIgURE 1: Comparison of computed and measured auto-ignition delay times versus 1000/T (K). Symbols: shock tube data [1] $\bigcirc$ : 13 bar $\Delta: 40$ bar; Solid Lines: detailed mechanism by Zhao et al. [10]; short dashed lines: 28-species reduced chemistry (this work); long dot-dashed lines: 26-species skeletal mechanism by Yao et al. $[14,15]$.

$900 \mathrm{~K}$, both the detailed and the 28-species mechanisms slightly overpredicted the auto-ignition delays while the 26species mechanism by Yao et al. $[14,15]$ grossly underpredicted the delays. Since the 26-species mechanism by Yao et al. $[14,15]$ was intended for engine conditions at high pressure and low temperature, the skeletal mechanism is not expected to work well outside the targeted combustion regime. Excellent agreement is shown between the present 28 -species chemistry (short dashed lines) and the detailed chemistry (long dot-dashed lines) [10] above $900 \mathrm{~K}$ as the lines are overlapping each other in this region. In addition, good agreement was found between the present 28species reduced chemistry and its parent mechanism when comparing computed delays under HCCI conditions (not shown).

3.2. Flame Propagation-Laminar Flame Speeds. The Sandia code PREMIX [21] was used to calculate the laminar flame speeds over a range of pressures ( $1 \mathrm{~atm}$ to $20 \mathrm{~atm}$ ) and equivalence ratios ( $\phi=0.6$ to 1.7$)$. For all runs, the initial temperature was set at $300 \mathrm{~K}$. The transport data provided by Zhao et al. [10] is used for these calculations. The 26-species skeletal mechanism by Yao et al. $[14,15]$ is not applicable to premixed combustion. Therefore, calculations were carried out only with the detailed mechanism by Zhao et al. [10] and the corresponding 28-species reduced chemistry. Figure 2 compares the computed flame speeds from these two mechanisms. Very good agreement can be seen between the two mechanisms over the wide range of conditions. For SAHCCI applications, the two mechanisms are also in excellent agreement (not shown) for the computed laminar flame speeds at elevated temperatures and pressures $(600 \mathrm{~K}$ to $750 \mathrm{~K}, 15$ bar to 20 bar).

3.3. Nonpremixed Flames. Simulations of counterflow, nonpremixed flames (Tsuji geometry) were carried out using 
the Sandia OPPDIF code [22] at ambient conditions and over a range of strain rates $a$, from $a=1 / s$ to $a=1000 / s$. A Tsuji flame geometry was chosen as a representative nonpremixed flame case due to its ability to be controlled by the strain rate. The fuel and oxidizer streams are composed of pure DME and pure air, respectively, both at 1 atm and $25^{\circ} \mathrm{C}$. Results were obtained only with the detailed DME mechanism [10] and the corresponding 28-species reduced chemistry. The computed flame extinction occurs around $a \sim 1250 / s$, with both mechanisms. Detailed comparisons of the calculated flame structures, including temperature and the mass fractions of $\mathrm{CO}$ and $\mathrm{H}$, are plotted versus mixture fraction in Figures 3, 4, and 5, respectively. All results were obtained at the following strain rates: $a=1 / s, 10 / s, 100 / s$, and $1000 / s$. The mixture fraction is based on the formulation proposed by Bilger [23] such that the stoichiometric value is independent of differential diffusion. The mixture fraction is defined as

$$
\xi=\frac{(1 / 2)\left(\left(Z_{H}-Z_{H, 0}\right) / M_{H}\right)+2\left(Z_{C}-Z_{C, 0}\right) / M_{C}-\left(Z_{O}-Z_{O, 0}\right) / M_{H}}{(1 / 2)\left(\left(Z_{H, 1}-Z_{H, 0}\right) / M_{H}\right)+2\left(Z_{C, 1}-Z_{C, 0}\right) / M_{C}-\left(Z_{O, 1}-Z_{O, 0}\right) / M_{O}},
$$

where $Z_{i}$ is the mass fraction of atomic species, $M_{i}$ is the corresponding atomic mass, and the subscripts 1 and 0 refer to the fuel and oxidizer streams, respectively.

As seen in Figure 3, the computed temperatures decrease with increasing strain rate. The peak temperature drops to roughly $1700 \mathrm{~K}$ at $a=1000 / s$ before extinction occurs. The corresponding CO levels are compared in Figure 4, showing the decrease in the peak $\mathrm{CO}$ value with increasing strain rate. Since CO is an intermediate species formed from decomposition of DME, CO decreases with decreasing residence time. Results of the predicted $\mathrm{H}$ radicals, presented in Figure 5, show an increase in the peak $H$ value with increasing strain rate. As the strain rate increases, the residence time decreases leading to less complete combustion. Results obtained with the 28-species reduced chemistry provide excellent agreement with results from the detailed mechanism. Similarly for diesel applications, good agreement is found in the computed flame structures between the detailed and reduced mechanisms for air at $900 \mathrm{~K}$ and 40 bar (not shown).

3.4. Applications to an HCCI Engine. The performance of the present 28-species reduced mechanism is further assessed through comparisons of numerical results, computed from 3D CFD simulations, against a new set of experimental data obtained under HCCI combustion. A brief description of the experiment is given below.

3.4.1. Experiment Setup with CFR Engine. Experiments were conducted using a single-cylinder ASTM-Cooperative Fuel Research (CFR) engine with an adjustable compression ratio. Detailed specifications of the engine and running conditions are listed in Table 3. Most notable of the conditions are the compression ratio, 8.26, and the operating RPM, 600, which were chosen to allow the engine to accommodate the range of tested equivalence ratios without approaching the misfiring or knocking regimes. It is also important to realize that while an operating RPM of 600 seems low, in fact this is an adequate testing RPM for the CFR engine as the maximum engine speed is 1,800 RPM and the normal RPM for RON testing is 600 RPM. Liquid DME was evaporated and then injected into intake port using a Quantum gaseous fuel injector. A Motec M4 ECU (Engine Control Unit) controlled injection timing, injection pulse width, and injection duty cycle. By varying the amount of fuel injected, the engine was run at three different equivalence ratios, $\phi=0.277,0.317$, and 0.358 . The equivalence ratio was determined from the conditions of the intake air and the mass of the fuel injected. The equivalence ratio was verified using Horiba analyzers for $\mathrm{O}_{2}$ and $\mathrm{CO}_{2}$. For each $\phi$, the compression ratio was set at 8.26 with steady HCCI operation. Cylinder pressure was measured using a 6052B Kistler piezoelectric pressure transducer and the signals were amplified by a 5044A Kistler charge amplifier. For each equivalence ratio, 300 cycles of incylinder pressure data were recorded at every 0.1 crank angle degree (CAD). The maximum measured NOx was around 23 ppm with an uncertainty of $1 \mathrm{ppm}$.

3.4.2. Data Analysis. A moving average filter, using three data points, was used to reduce noise in the pressure data. The net heat release rate was calculated on the basis of first law of thermodynamics as [24]

$$
\frac{d Q}{d \theta}=\frac{\gamma}{\gamma-1} P \frac{d V}{d \theta}+\frac{1}{\gamma-1} V \frac{d P}{d \theta}
$$

where $Q$ is the net heat release rate, $\theta$ the crank angle, $V$ the cylinder volume, and $\gamma$ the heat capacity ratio. Instead of using a fixed value for $\gamma$, its variation with crank angle is estimated as follows. The values of $\gamma(\theta)$ were assumed constant only during certain periods of the compression and expansion stokes, defining $\gamma(\theta)$ as $\gamma_{\text {comp }}$ and $\gamma_{\text {exp }}$, respectively, during these periods. During the compression stroke, isentropic compression was assumed from - 120 CAD to $-40 \mathrm{CAD}$ after top dead center (ATDC). Using the isentropic relationship $P V^{\gamma_{\text {comp }}}=$ constant, the value of $\gamma_{\text {comp }}$ is computed from pressure data and cylinder volumes at $\theta=-120 \mathrm{CAD}$ and $\theta=-40 \mathrm{CAD}$ ATDC. Similarly, the value of $\gamma_{\exp }$ is determined for the expansion stroke from $60 \mathrm{CAD}$ to $120 \mathrm{CAD}$ ATDC. Between $-40 \mathrm{CAD}$ and $60 \mathrm{CAD}$ ATDC, $\gamma$ was assumed to vary linearly as

$$
\gamma(\theta)=\gamma_{\mathrm{comp}}+\frac{\theta+40}{100\left(\gamma_{\exp }-\gamma_{\mathrm{comp}}\right)}
$$




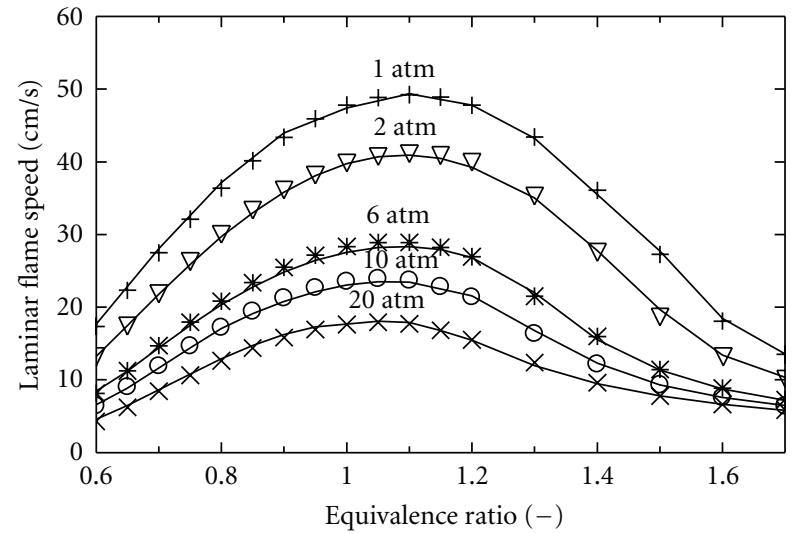

FIGURE 2: Comparison of predicted laminar flame speeds versus equivalence ratio at various pressures ( $1 \mathrm{~atm}, 2 \mathrm{~atm}, 6 \mathrm{~atm}, 10 \mathrm{~atm}$, and $20 \mathrm{~atm})$. Solid lines: detailed DME mechanism by Zhao et al. [10]; symbols: 28-species reduced chemistry (this work). Initial temperature: $300 \mathrm{~K}$.

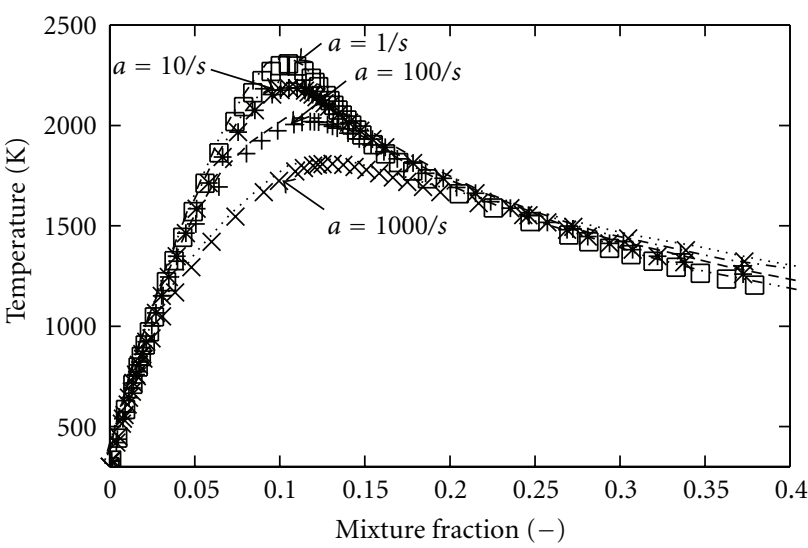

FIGURE 3: Comparison of computed temperatures versus mixture fraction for a Tsuji burner at strain rates $a=1 / s, 10 / s, 100 / s, 1000 / s$. Lines: detailed DME chemistry by Zhao et al. [10]; symbols: 28species reduced chemistry (this work).

TABLE 3: Operating conditions for the pressure trace simulation of the CFR engine.

\begin{tabular}{lc}
\hline Fuel & Dimethyl ether \\
\hline Bore & $8.255 \mathrm{~cm}$ \\
Stroke & $11.43 \mathrm{~cm}$ \\
Compression Ratio & 8.26 \\
Intake Pressure & $1 \mathrm{bar}$ \\
Intake Temperature & $298 \mathrm{~K}$ \\
Engine Coolant Temperature & $348 \mathrm{~K}$ \\
Equivalence Ratios & $0.277,0.317,0.358$ \\
Engine Speed & $600 \mathrm{RPM}$ \\
\hline
\end{tabular}

The cumulative net heat release was calculated by integrating (2) with respect to crank angle, $\theta$, from -120 CAD to 60 CAD ATDC.

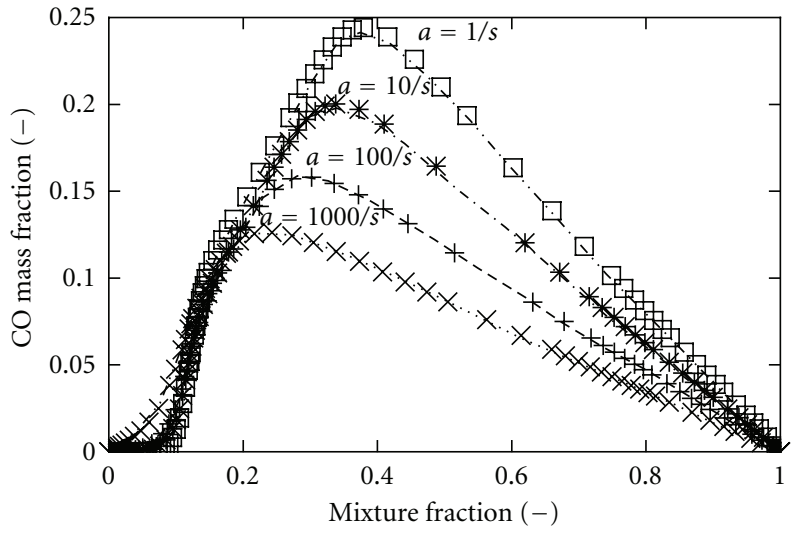

Figure 4: Comparison of computed CO mass fractions versus mixture fraction for a Tsuji burner at strain rates $a=1 / s, 10 / s$, 100/s, 1000/s. Lines: detailed DME chemistry by Zhao et al. [10]; symbols: 28-species reduced chemistry (this work).

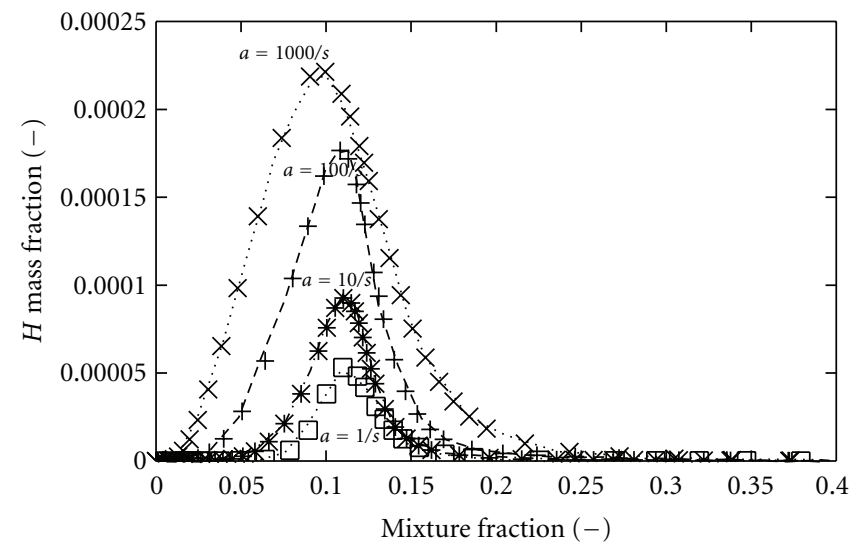

Figure 5: Comparison of computed $\mathrm{H}$ mass fractions versus mixture fraction for a Tsuji burner at strain rates $a=1 / s$.

An error analysis was performed on all presented data. The details of the error analysis are presented in the supplementary materials.

3.4.3. Three-Dimensional Simulations. Three-dimensional (3D) CFD simulations were carried out using a modified version of KIVA-3V [25] with both the detailed and the 28species reduced chemistry. The modified version of KIVA-3V has been used in a number of prior publications, including the paper by Tham et al. [17]. The 3-D grid consists of 53, 616 cells at bottom dead center and 17, 040 cells at top dead center. The simulations were carried between -180 CAD ATDC, at bottom dead center, and 50 CAD ATDC only. As the simulation was started at -180 CAD ATDC, the temperature at bottom dead center was estimated from the engine intake temperature. Crevices and blowby effect were not included in the simulations. Therefore, the Compression Ratio (CR) of the simulations was set at $\mathrm{CR}=7.66$, matching the computed motoring pressure traces to the experimental data (not shown). The uncertainty 
TABLE 4: Initial conditions at bottom dead center used in 3-D CFD engine simulations.

\begin{tabular}{lccccccc}
\hline $\begin{array}{l}\text { Equivalence } \\
\text { ratio }(\phi)\end{array}$ & $\begin{array}{c}\text { Temp. } \\
(\mathrm{K})\end{array}$ & $\begin{array}{c}\text { Pressure } \\
\text { (bar) }\end{array}$ & $\begin{array}{c}\mathrm{CH}_{3} \mathrm{OCH}_{3} \\
\text { (mass fraction) }\end{array}$ & $\begin{array}{c}\mathrm{O}_{2} \\
\text { (mass fraction) }\end{array}$ & $\begin{array}{c}\mathrm{H}_{2} \mathrm{O} \\
\text { (mass fraction) }\end{array}$ & $\begin{array}{c}\mathrm{CO}_{2} \\
\text { (mass fraction) }\end{array}$ & $\begin{array}{c}\mathrm{N}_{2} \\
\text { (mass fraction) }\end{array}$ \\
\hline 0.277 & 345 & 1 & 0.02744 & 0.2208 & 0.003013 & 0.004283 & 0.7445 \\
0.317 & 345 & 1 & 0.03175 & 0.2194 & 0.003109 & 0.005063 & 0.7407 \\
0.358 & 345 & 1 & 0.03557 & 0.2179 & 0.003385 & 0.005512 & 0.7380 \\
\hline
\end{tabular}

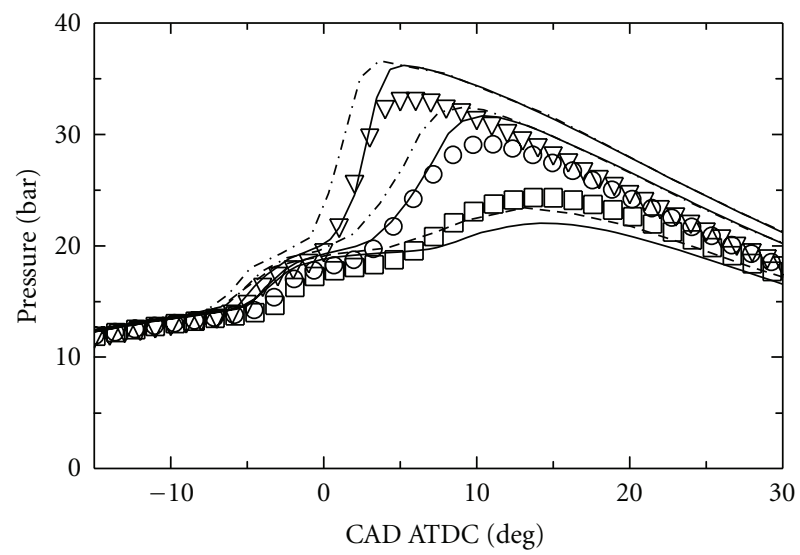

FIGURE 6: Comparison of cylinder pressure traces versus crank angle degree after top dead center (CAD ATDC). Symbols: experimental data from a CFR engine with different equivalence ratios $(\phi): \nabla$ $(\phi=0.358) ; \bigcirc(\phi=0.317) ; \square(\phi=0.277)$; Solid lines: detailed DME chemistry [10]; Dashed lines: 28-species reduced chemistry (this work).

of the pressure and $\mathrm{CAD}$ measurements during engine motoring was found to be 0.2 bar and $0.05 \mathrm{CAD}$, respectively. Initial conditions at -180 CAD ATDC are listed in Table 4. The initial species compositions were estimated by running a single-zone reactor model over several cycles so that the residual gases are properly represented in the unburned mixture. The single zone reactor estimated the temperature at the bottom dead center to be $315 \mathrm{~K}$; however, preliminary 3-D simulations with initial temperature set at $315 \mathrm{~K}$ failed to autoignite. Therefore, the initial temperature for the 3D simulations was increased to $345 \mathrm{~K}$, in order to roughly match the predicted Start of Combustion (SOC) to the experimental data.

3.4.4. Pressure Trace. Figure 6 compares the computed pressure traces from the detailed mechanism by Zhao et al. [10] and the 28-species chemical mechanisms against the CFR data at three equivalence ratios $(\phi=0.277,0.317$, and 0.358). During engine firing, the maximum uncertainty of the pressure across all three equivalence ratios was found to be 0.3 bar. As the combustible mixture gets leaner, the SOC is seen to retard, as expected. Under the present engine conditions, DME starts to combust at low temperatures $(\sim 700 \mathrm{~K})$ with a large amount of heat release prior to the main auto-ignition $(\sim 1000 \mathrm{~K})$, a feature unique to DME combustion. Overall, the numerical model correctly predicts the effect of equivalence ratio on SOC, which is in good

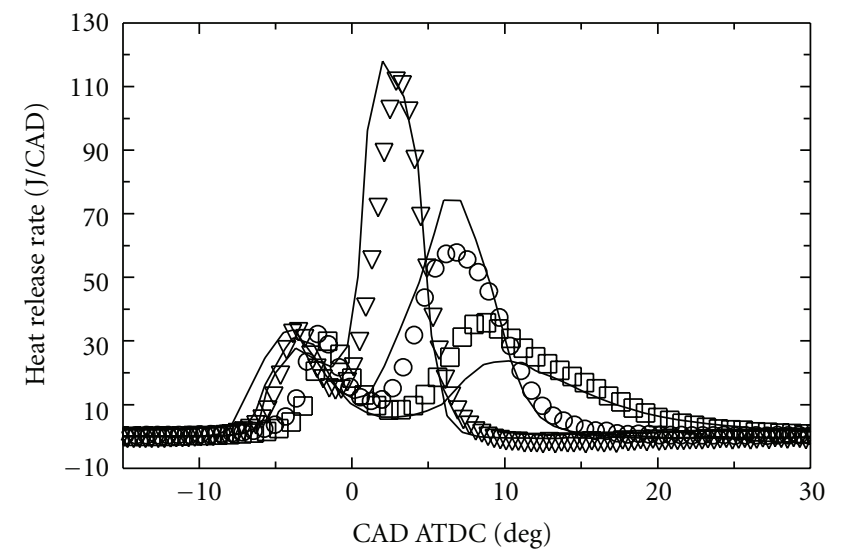

Figure 7: Comparison of heat release rates versus crank angle degree after top dead center (CAD ATDC). Symbols: experimental data from a CFR engine with different equivalence ratios $(\phi): \nabla$ $(\phi=0.358) ; \bigcirc(\phi=0.317) ; \square(\phi=0.277)$; Lines: detailed DME chemistry [10].

agreement with the data. Although the initial temperature was set $30 \mathrm{~K}$ higher than estimated, the amount of low temperature heat release is well predicted.

Figure 6 also shows reasonable agreement between the 28 -species reduced chemistry and the detailed mechanism [10]. With the present 28-species reduced chemistry, the CPU time for 3-D simulations is about one sixth of that used by the detailed mechanism. However, the reduced chemistry predicts an earlier SOC relative to the detailed chemistry. This discrepancy is consistent with the results shown in Figure 1, which reveal slightly shorter predicted auto-ignition delay times for the reduced chemistry at both 13 bar and 40 bar.

3.4.5. Heat Release Rate. Figures 7 and 8 compare the heat release rates obtained from the CFR engine, the detailed chemistry, and the 28-species reduced chemistry model. The maximum uncertainty of the computed net heat release rate was found to be $9.5 \mathrm{~J} / \mathrm{CAD}, 19 \mathrm{~J} / \mathrm{CAD}$, and $41 \mathrm{~J} / \mathrm{CAD}$ for the $0.277,0.317$, and 0.377 equivalence ratios, respectively. Compared to the experimental data, the results from both detailed and reduced chemistry show a later start of lowtemperature heat release, a lower peak heat release rate for $\phi=0.317$ and 0.358 , and a higher peak heat release rate for $\phi=0.277$. Additionally, the duration of the lowtemperature heat release is longer for the 28-species case, while the duration of the low-temperature heat release for the detailed mechanism is in good agreement with the data. 


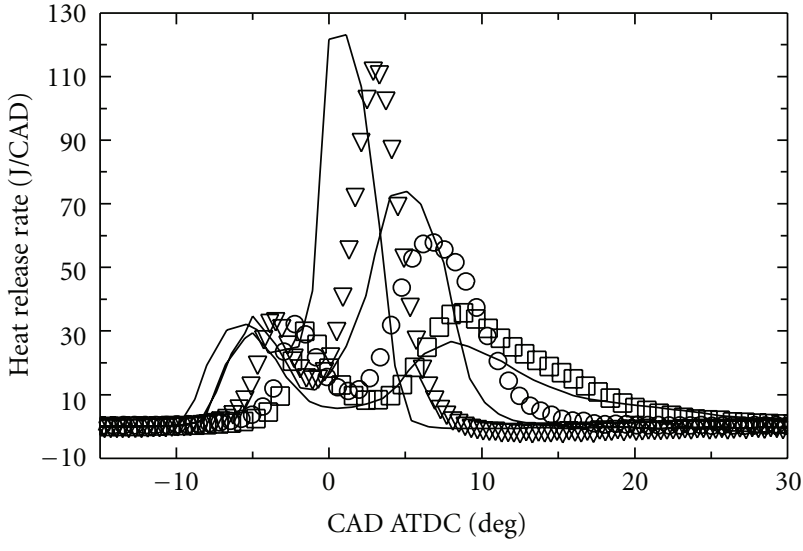

Figure 8: Comparison of heat release rates versus crank angle degree after top dead center (CAD ATDC). Symbols: experimental data from a CFR engine with different equivalence ratios $(\phi): \nabla$ ( $\phi=0.358) ; \bigcirc(\phi=0.317) ; \square(\phi=0.277)$; Lines: 28 -species reduced DME chemistry (this work).

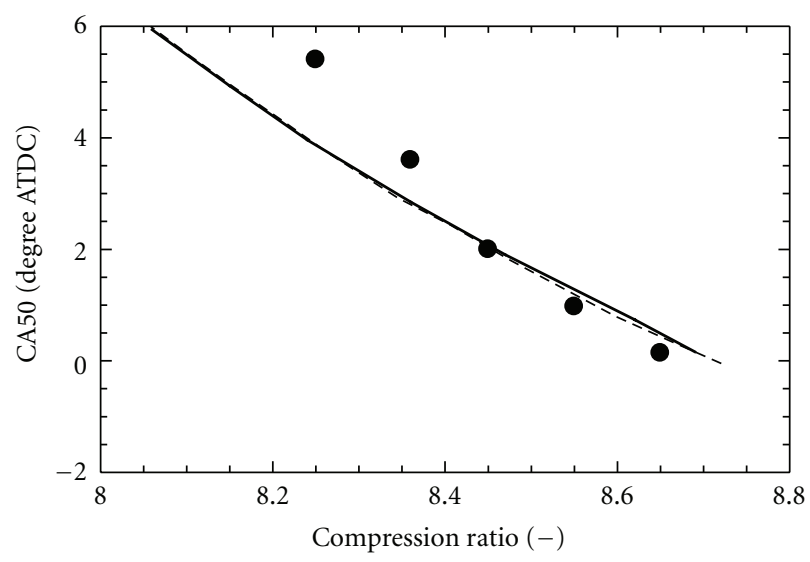

FIGURE 9: Comparison of CA50 versus compression ratio (CR): Symbols: data taken from CFR with $(\phi=0.33)$ and ambient temperature and pressure; Solid lines: single zone model using detailed DME chemistry [10] with $T_{0}=305 \mathrm{~K}$; dashed lines: single zone model 28-species reduced DME chemistry with $T_{0}=314 \mathrm{~K}$ (this work).

The overprediction of the auto-ignition delay, as mentioned in the previous section (Figure 1), is consistent with the later start of the low-temperature heat release for both numerical cases.

3.4.6. Sensitivity of SOC to Compression Ratio. Experimental runs were conducted to explore the sensitivity of SOC on CR at $\phi=0.33$, while keeping all other operating parameters the same as before. The SOC is conventionally characterized by the CA50, defined as the crank-angle degree at which $50 \%$ of the cumulative heat release occurs. Such exploration runs provide additional evaluation of the chemistry model. Corresponding numerical simulations were conducted using a single zone reactor with both the 28-species reduced chemistry and its detailed parent mechanism [10]. The initial engine temperatures were chosen simply to align the curves as the slopes of these curves, indicative of the sensitivity of SOC to compression ratio, are more important for this study than the actual values of CA50. Figure 9 compares the measured and predicted CA50 versus the CR, showing consistently that increasing CR decreases CA50. The experimental results reveal that the SOC of DME is very sensitive to CR. By increasing CR from 8.25 to 8.65 (a mere 5\% increase), CA50 decreases by 6 degrees. In comparison to the data, both mechanisms predicted slightly less sensitivity of SOC on CR; the predicted CA50 decreases by 4 degrees when CR is increased from 8.25 to 8.65 . The maximum uncertainty of the computed value for CA50 was found to be $1.2,0.8$, and $0.3 \mathrm{CAD}$ for equivalence ratios of $0.277,0.317$, and 0.377 respectively.

\section{Conclusions}

Considerable research has been conducted in the past to construct detailed and simplified DME chemical mechanisms for use in numerical simulations. In the present study, a 28-species reduced chemical mechanism was developed to cover a wide range of engine conditions anticipated in future advanced engines, such as HCCI, SAHCCI, and PCCI.

The performance of the reduced chemistry is found to be satisfactory to the following combustion modes after comparing computed auto-ignition delays, laminar flame speeds, and non-premixed flame structures against those from the detailed mechanism by Zhao et al. [10]. The reduced mechanism was also used in 3-D CFD simulations and the predicted pressure traces and engine heat release rates were compared with in-house CFR engine data at three different equivalence ratios. The detailed and reduced mechanisms predicted the effect of equivalence ratio on SOC in good agreement with experimental data. However, the initial bottom dead center temperature of the numerical model needed to be set $30 \mathrm{~K}$ degrees higher than estimated in order to match SOC from the CFR engine. In addition, the detailed and reduced mechanisms predicted an earlier start of ignition than the CFR engine data. Additional exploration of the effect of CR on SOC suggests both the detailed and reduced mechanisms under-predict the sensitivity of SOC with CR. It is important to stress that while the level of agreement seen with the engine cases does not match the level of agreement seen with the other flame cases and that additional species and reactions could have been added to the reduced chemistry to improve its accuracy, the goal of these validations is to simply verify the effectiveness of the 28-species DME reduced mechanism which had been generated from an automatic, verified process [17-19]. Overall, the successful development of the present 28-species reduced chemistry mechanism confirms the effectiveness of recently developed strategies in developing reduced chemistry for use in a wide range of combustion regimes. However, future research is required for further refinement of the detailed DME mechanism by Zhao et al. [10] in order to provide more accurate HCCI engine simulations. 


\section{References}

[1] C. Arcoumanis, C. Bae, R. Crookes, and E. Kinoshita, "The potential of di-methyl ether (DME) as an alternative fuel for compression-ignition engines: a review," Fuel, vol. 87, no. 7, pp. 1014-1030, 2008.

[2] J. Song, Z. Huang, X. Qiao, and W. Wang, "Performance of a controllable premixed combustion engine fueled with dimethyl ether," Energy Conversion and Management, vol. 45, no. 13-14, pp. 2223-2232, 2004.

[3] U. Pfahl, K. Fieweger, and G. Adomeit, "Self-ignition of dieselrelevant hydrocarbon-air mixtures under engine conditions," Proceedings of the Combustion Institute, vol. 26, no. 1, pp. 781789, 1996.

[4] P. Dagaut, J. C. Boettner, and M. Cathonnet, "Chemical kinetic study of dimethylether oxidation in a jet stirred reactor from 1 to 10 ATM: experiments and kinetic modeling," Proceedings of the Combustion Institute, vol. 26, no. 1, pp. 627-632, 1996.

[5] H. J. Curran, W. J. Pitz, C. K. Westbrook, P. Dagaut, J. C. Boettner, and M. Cathonnet, "A wide range modeling study of dimethyl ether oxidation," International Journal of Chemical Kinetics, vol. 30, no. 3, pp. 229-241, 1998.

[6] P. Dagaut, C. Daly, J. M. Simmie, and M. Cathonnet, "Oxidation and ignition of dimethylether from low to high temperature (500-1600 K): experiments and kinetic modeling," Proceedings of the Combustion Institute, vol. 1, pp. 361369, 1998.

[7] S. L. Fischer, F. L. Dryer, and H. J. Curran, "Reaction kinetics of dimethyl ether. I: high-temperature pyrolysis and oxidation in flow reactors," International Journal of Chemical Kinetics, vol. 32, no. 12, pp. 713-740, 2000.

[8] H. J. Curran, S. L. Fischer, and F. L. Dryer, "Reaction kinetics of dimethyl ether. II: low-temperature oxidation in flow reactors," International Journal of Chemical Kinetics, vol. 32, no. 12, pp. 741-759, 2000.

[9] E. W. Kaiser, T. J. Wallington, M. D. Hurley et al., "Experimental and modeling study of premixed atmospheric-pressure dimethyl ether-air flames," Journal of Physical Chemistry A, vol. 104, no. 35, pp. 8194-8206, 2000.

[10] Z. Zhao, M. Chaos, A. Kazakov, and F. L. Dryer, "Thermal decomposition reaction and a comprehensive kinetic model of dimethyl ether," International Journal of Chemical Kinetics, vol. 40, no. 1, pp. 1-18, 2008.

[11] H. Yamada, H. Sakanashi, N. Choi, and A. Tezaki, "Simplified oxidation mechanism of DME applicable for compression ignition," SAE Paper 2003-01-1819, 2003.

[12] X. Liang, Numerical study on HCCI combustion of DME/ $\mathrm{MEOH}$ dual fuels, Master's Dissertation, Tianjin University, Tianjin, China, 2005.

[13] M. Yao, J. Qin, and Z. Zheng, "Numerical study of the combustion mechanism of a homogeneous charge compression ignition engine fuelled with dimethyl ether and methane, with a detailed kinetics model. Part 1: the reaction kinetics of dimethyl ether," Proceedings of the Institution of Mechanical Engineers, Part D: Journal of Automobile Engineering, vol. 219, no. 10, pp. 1213-1223, 2005.

[14] X. Liang, M. F. Yao, and Z. Q. Zheng, "Numerical study on the mechanism of low temperature reaction of DME/methanol HCCI combustion," Journal of Combustion Science and Technology, vol. 11, no. 2, pp. 149-154, 2005.

[15] M. Yao, C. Huang, and Z. Zheng, "Multidimensional numerical simulation on dimethyl ether/methanol dual-fuel homogeneous charge compression ignition (HCCI) engine combustion and emission processes," Energy and Fuels, vol. 21, no. 2, pp. 812-821, 2007.

[16] C. Huang, M. Yao, X. Lu, and Z. Huang, "Study of dimethyl ether homogenous charge compression ignition combustion process using a multi-dimensional computational fluid dynamics model," International Journal of Thermal Sciences, vol. 48, pp. 1814-1822, 2009.

[17] Y. F. Tham, F. Bisetti, and J. Y. Chen, "Development of a highly reduced mechanism for iso-octane HCCI combustion with targeted search algorithm," Journal of Engineering for Gas Turbines and Power, vol. 130, no. 4, Article ID 042804, 2008.

[18] J. Y. Chen, "Automatic generation of reduced mechanisms and their applications to combustion modeling," Transactions of the Aeronautical and Astronautical Society of the Republic of China, vol. 33, no. 2, pp. 59-67, 2001.

[19] J. Y. Chen and Y. F. Tham, "Speedy solution of quasi-steadystate species by combination of fixed-point iteration and matrix inversion," Combustion and Flame, vol. 153, no. 4, pp. 634-646, 2008.

[20] A. E. Lutz, R. J. Kee, and J. A. Miller, "SENKIN: a fortran program for predicting homogeneous gas phase chemical kinetics with sensitivity analysis," Tech. Rep. SAND87-8248, Sandia National Laboratories, Albuquerque, NM, USA, 1988.

[21] R. J. Kee, J. F. Grcar, M. D. Smooke, and J. A. Miller, "PREMIX: a Fortran program for modeling steady, laminar, one-dimensional premixed flames," Tech. Rep. SAND85-8240, Sandia National Laboratories, Albuquerque, NM, USA, 1985.

[22] A. E. Lutz, R. J. Kee, J. F. Grcar, and F. M. Rupley, “OPPDIF: a Fortran program for computing opposed-flow diffusion flames," Tech. Rep. SAND96-8243, Sandia National Laboratories, Albuquerque, NM, USA, 1985.

[23] R. W. Bilger, "Structure of turbulent nonpremixed flames," Proceedings of the Combustion Institute, vol. 22, pp. 475-488, 1988.

[24] J. B. Heywood, Internal Combustion Engine Fundamentals, McGraw-Hill, New York, NY, USA, 1988.

[25] "KIVA3V: a block structured KIVA program for engines with vertical or canted valves," Tech. Rep. LA-13313-MS, Los Alamos Laboratory, Los Alamos, NM, USA, 1997. 

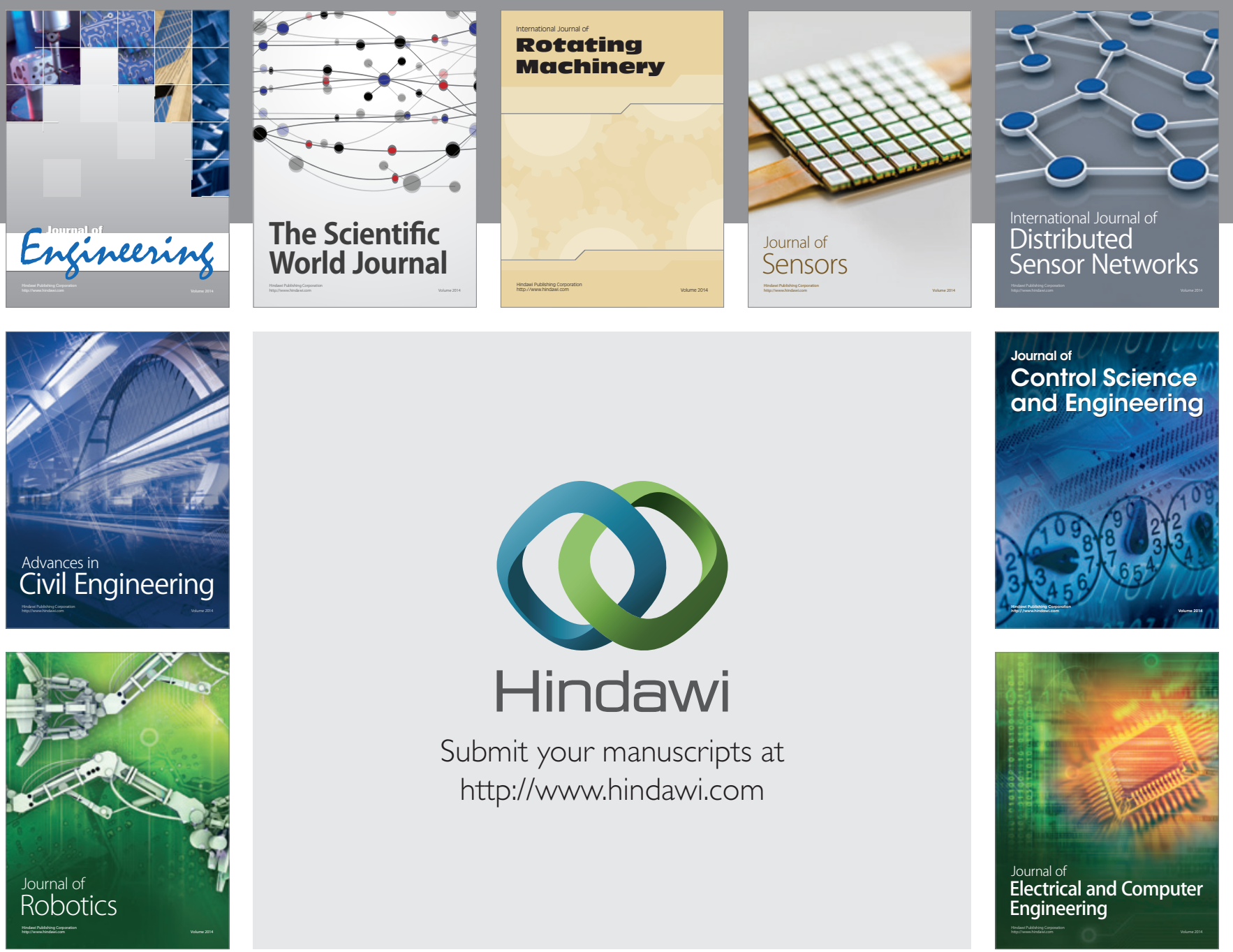

Submit your manuscripts at

http://www.hindawi.com
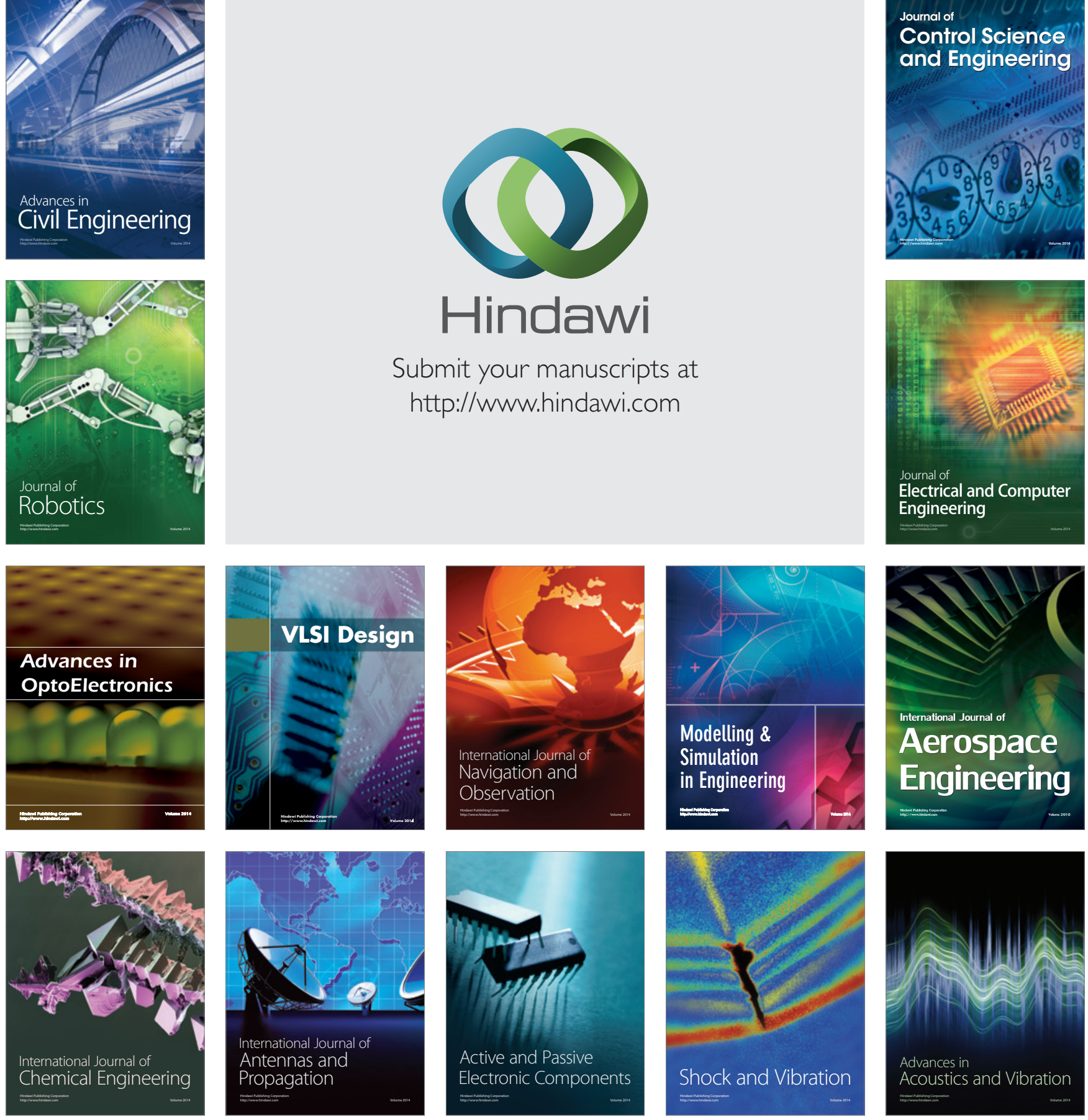